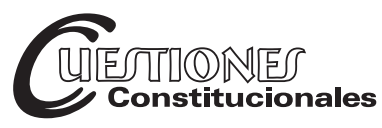

Revista Mexicana de Derecho Constitucional

Núm. 39, julio-diciembre 2018

\title{
Derecho de acceso a la información entre la Constitución y la realidad
}

\author{
The Right to Access to Public Information \\ in the Mexican Constitution
}

\begin{abstract}
RESUMEN: Este artículo explora la brecha entre la enunciación constitucional del derecho de acceso a la información pública y el ejercicio efectivo del mismo a través de los portales de transparencia de las autoridades mexicanas. Busca evidenciar la necesidad de transitar de los portales de transparencia para sólo cumplir con las leyes (de jure) a herramientas de una política de transparencia proactiva que permitan la interacción entre la autoridad y las personas para el cabal ejercicio de sus derechos (de facto). Para ello se pregunta cuál es el objetivo de un portal de transparencia y qué características debiera tener la información en él presentada. Al efecto se hace una revisión de la literatura respecto de la calidad de la información pública en portales de transparencia, se analiza la situación de México para reflexionar sobre las áreas de oportunidad que potencien el uso de los portales de acceso a la información, la libertad de expresión y su ejercicio efectivo.
\end{abstract}

Palabras clave: derecho de acceso a la información, libertad de expresión, transparencia, información pública de oficio, portales de transparencia, gobierno abierto, datos abiertos.

* Doctora en derecho por la UNAM; maestra en derecho por la Universidad de Georgia, Estados Unidos; maestra en filosofía por la Universidad del Mayab y licenciada en derecho por el ITAM. Es coordinadora de la Maestría en Administración y Políticas Públicas y profesora en el CIDE.ana.fierro@cide.edu. ment of the rights of freedom of speech and access to public information in the Mexican Constitution. It analyzes the gap between the de jure recognition in the Constitution of these rights and the de facto access to useful and reusable information for the exercise of human rights on the other. It seeks to answer what is the purpose of the information posted in the government websites, and what should be its quality in order to guarantee the right to access of information in an era of open government. For that purpose it reviews the literature on the quality of public information and explores the areas of opportunity of the Mexican government websites.

Keywords: Access to Information Rights, Freedom of Speech, Transparency, Public Information, Transparency Websites, Open Government, Open Data. 
Sumario: I. Introducción. II. Objetivo de un portal de transparencia. III. Portales de transparencia en México. IV. Retos y oportunidades. V. Reflexiones finales. VI. Bibliografía.

\section{INTRODUCCIÓN}

Uno de los avances más importantes del constitucionalismo mexicano en el siglo XXI ha sido el reconocimiento y consolidación del derecho humano de acceso a la información pública como elemento del cabal ejercicio de la libertad de expresión. ${ }^{1}$ Hoy en día contamos con un marco legal que, a partir del artículo 6o. constitucional y la reciente emisión de la Ley General de Transparencia LGT, garantiza que toda persona pueda conocer a los sujetos obligados de los tres niveles de gobierno y de los tres poderes, cuáles son sus principales funciones, de qué recursos públicos disponen y cómo los ejercen. Asimismo, durante buena parte de este siglo tanto en la Federación como en las entidades federativas se han emitido diversas leyes que buscan detallar los contenidos constitucionales de modo que se satisfaga a plenitud el derecho de acceso a la información pública. Sin embargo, las políticas públicas de transparencia que debieran derivar del artículo 6o. constitucional se han quedado atrás.

Tal circunstancia no es exclusiva del derecho humano de acceso a la información. Por el contrario, Law y Versteeg en un estudio sobre efectividad de las Constituciones en el mundo señalan que, aunque en la actualidad existen importantes movimientos que han empujado a los Estados a reconocer en sus textos constitucionales más derechos humanos, la cantidad de estos derechos que en la práctica realmente son efectivos crece a una tasa mucho menor. De manera que la distancia entre los derechos constitucionales de jure y su goce y ejercicio efectivo de facto aún es importante. Existen Constituciones con un listado escueto de derechos que presentan un alto

Así lo ha señalado la Comisión Interamericana de Derechos Humanos, en la Declaración de Principios sobre la Libertad de Expresión adoptada en 2000, de la que destaca: "El acceso a la información en poder del Estado es un derecho fundamental de los individuos. Los Estados están obligados a garantizar el ejercicio de este derecho. Este principio sólo admite limitaciones excepcionales que deben estar establecidas previamente por la ley para el caso que exista un peligro real e inminente que amenace la seguridad nacional en sociedades democráticas". CIDH, Informe Anual 2000-01, vol. III, p. 135. 
índice de efectividad como en Finlandia, Eslovenia, Suiza, España, Chile, Suecia, Nueva Zelanda y Uruguay, y Constituciones con un extenso listado de derechos que no logran implementarse como son los casos de Nigeria, Myanmar, Sudán, Rusia, Vietnam, Congo y Afganistán. De acuerdo con este estudio, el caso de México se encuentra en el promedio. ${ }^{2}$ Sin embargo, el derecho humano al acceso a la información es justo el ejemplo en el que si bien su incorporación al texto constitucional en 2007, las subsecuentes reformas al artículo 6o. en 2014 y las leyes generales, federales y locales mucho han avanzado en la consolidación de este derecho humano de jure, hay una distancia importante aún con la implementación de los procesos y la concientización de los servidores públicos sobre la necesidad de contar con una política de transparencia acorde con las exigencias del texto constitucional.

Uno de los aspectos donde la brecha entre la norma y la realidad es mayor, son los portales de transparencia. Hoy los sujetos obligados los utilizan como un simple depósito de información para cumplir formalmente con la ley. Esta visión de un mero cumplimiento formal respecto de la información pública de oficio resulta insuficiente para el eficaz ejercicio de los derechos de acceso a la información y libertad de expresión. Tras 10 años de avances de la sociedad civil mexicana, la consolidación de los derechos humanos a través de la reforma al artículo 1o. constitucional en 2011 y en consonancia con lo que sucede en otros países, ya no basta con publicar la información que la ley mandata, sino que la calidad de la misma también importa.

Adicionalmente, resultado de regulación internacional en la materia como la Alianza para el Gobierno Abierto (AGA), del que México es parte desde 2011, se exige a los Estados que la información pública cumpla con estándares de calidad internacionales, que permitan a los ciudadanos no sólo conocer, sino comprender y reutilizar la información pública de manera que sea un medio para recibir mejores servicios públicos, exigir sus derechos y participar en la toma de decisiones públicas. ${ }^{3}$ Ello requiere de implementar una política de transparencia proactiva que vea en el ciudadano no sólo un receptor pasivo de información pública, sino un usuario de la información para el ejercicio de sus derechos incluyendo los que se refieren a los servi-

2 Law, David y Versteeg, Mila, Sham Constitutions, 101 Cal. L. Rev. 863, 2013, disponible en: http://scholarship.law.berkeley.edu/californialawreview/vol101/iss4/.

3 Disponible en: http://www.opengovpartnership.org/es. 
cios que debe prestarles el Estado, así como para participar activamente de la rendición de cuentas y de la toma de decisiones públicas.

En tal sentido, este artículo explora la brecha entre la enunciación constitucional del derecho de acceso a la información pública y el ejercicio efectivo del mismo a través del caso de los portales de transparencia que toda autoridad en los tres niveles de gobierno debe tener. Con ello busca poner de manifiesto la necesidad de transitar de la visión de los portales de transparencia como meros receptáculos de información para cumplir con las leyes de transparencia (de jure) a herramientas de una política de transparencia proactiva que permite la interacción entre la autoridad y las personas para el cabal ejercicio de sus derechos (de facto). Para ello la pregunta a resolver es cuál es el objetivo de un portal de transparencia y qué característica debiera tener la información en él presentada para cumplirla.

Al efecto se hace un revisión de la literatura respecto de la calidad de la información pública en portales de transparencia de diferentes países, se analiza la situación de México y los resultados arrojados por estudios como la Métrica de Transparencia 2014, ${ }^{4}$ para finalmente reflexionar sobre las áreas de oportunidad que a partir del artículo 6o. constitucional, la Ley General de Transparencia y su subsecuente implementación, tanto en la Federación como en los estados debieran aprovecharse para potenciar el uso de los portales de transparencia en cuanto herramientas de comunicación que permitan realmente reducir la brecha entre la formalización del derecho humano de acceso a la información y su ejercicio efectivo.

\section{OBJETIVO DE UN PORTAL DE TRANSPARENCIA}

El primer reto al determinar el objetivo de un portal de transparencia es conceptualizar la transparencia. La academia la entiende en un sentido amplio como el flujo de información en una polis, ${ }^{5}$ el nivel necesario de

\footnotetext{
$4 \quad$ Disponible en: $h t t p: / / w w w . m e t r i c a d e t r a n s p a r e n c i a . c i d e . e d u$.

5 Rosendorff, Peter et al., Measuring transparency, Department of Political Science, University of Minnesota, 2013, disponible en: http://papers.ssrn.com/sol3/papers. cfm?abstract_id=2113665.
} 
publicación de información para una adecuada representación; ${ }^{6}$ o bien, mediante definiciones más acotadas que la entienden como la publicación de la información relevante para la evaluación de las autoridades, ${ }^{7}$ o incluso como la presentación de información para rendir cuentas. ${ }^{8}$ Sin duda, la publicación de información como primer paso de la evaluación del quehacer de las autoridades y prerrequisito para rendir cuentas constituye una de las razones principales de la transparencia, entendida como correlativo de la libertad de expresión.

Sin embargo, una vez que se tiene acceso a la información es necesario que se pueda utilizar para exigirle cuentas a las autoridades, tomar decisiones informadas respecto a quienes elegimos como representantes, participar en las decisiones públicas o conocer cómo y dónde ejercer nuestros derechos o recibir servicios públicos. De manera tal, que entonces ya no basta con que se publique información, sino que los formatos y el lenguaje en la que ésta se hace accesible también importan. Aunque el uso de Internet para divulgar información es ya una práctica común en el orbe y conforme al inciso B del artículo 6o. constitucional es un derecho humano, análisis han demostrado que el uso de portales de transparencia tendrá muy poco valor social si los ciudadanos no pueden comprender y reutilizar esta información para ejercer sus derechos, recibir mejores servicios públicos o participar en la toma de decisiones públicas; ${ }^{9}$ además, si los portales no son percibidos como herramientas que apoyan el quehacer público, entonces son desaprovechados por los propios órganos del Estado que los consideran sólo un requisito formal adicional para cumplir con la ley. ${ }^{10}$ De tal manera que al deber de hacer accesible la información pública, hay que añadir la calidad de la información como elemento esencial que

6 Schnackenberg, Andrew, Measuring Transparency: Towards a Greater Understanding of Systemic Transparence and Accountability, 2002.

7 Bauhr, Monica y Grimes, Marcia, What is Government Transparency?, Quality of Government Institute, working paper series, 2012, p. 16.

8 López-Ayllón, Sergio, El acceso a la información como un derecho fundamental: la reforma al artículo 6o. de la Constitución mexicana, México, Instituto Federal de Acceso a la Información Pública, 2009.

9 Kraemer, Kenneth L. y King John, Leslie, International Journal of Electronic Government Research, vol. 2, núm. 1, 2006, pp. 1-20.

10 Fierro, Ana Elena y Gil-García, J. Ramón, Más allá del acceso a la información El uso de tecnologías de información para fomentar la transparencia, la participación y la colaboración en el sector público, en Cejudo, Guillermo et al. (eds.), La política de transparencia en México, México, CIDE, 2012, pp. 207-239. 
permite no sólo el cumplimiento formal de la ley, sino que también busca que la información sea útil y de interés para los ciudadanos. ${ }^{11}$ Así, a partir de iniciativas como AGA se establece que los gobiernos no sólo deben ser transparentes, sino que deben rendir cuentas y escuchar las exigencias de los ciudadanos respecto de la mejora en los servicios públicos, el ejercicio de los derechos y la participación ciudadana. ${ }^{12}$ Hoy, la encomienda ya no está limitada a publicar información conforme a un mandato legal, sino que se convierte en un intercambio de datos y opiniones entre gobernantes y gobernados. La finalidad del AGA es lograr fomentar la colaboración dentro del gobierno y la participación ciudadana en la toma de decisiones públicas. ${ }^{13}$ Además, como producto de este acuerdo bilateral tiene una dimensión transfronteriza al intercambio de información guiado por la accesibilidad de los datos y el fomento de la colaboración de manera que el uso del saber colectivo permita la participación ciudadana y la mejor toma de decisiones públicas con una visión de universalidad.

Hoy los gobiernos no sólo deben presentar la información señalada en las leyes de transparencia, sino que también deben garantizar que ésta sea accesible tanto en el formato como en el lenguaje; que sea útil, y cuando proceda que tenga formatos de datos abiertos que permitan su reutilización. De tal manera que todas las personas, sin importar su nacionalidad o localización geográfica, puedan contar con información estratégica ${ }^{14}$ que les permita interactuar de manera efectiva con la autoridad, además de dotarlos de las herramientas para ejercer sus derechos y participar en la toma de decisiones públicas. Ello conforma una política de transparencia proactiva donde el Internet se convierte en una poderosa herramienta que permite una interacción directa entre la sociedad y los órganos del Estado, sin intermediarios y que incluso reduce costos de transacción respecto tanto del acceso a la información como del ejercicio de derechos y la participación ciudadana. ${ }^{15}$ Así, ya no basta con que los gobiernos presenten un cúmulo

11 Cucciniello, Maria y Nasi, Greta, Making Transparency Transparent: an Assessment Model for Local Governments, Reforming the Public Sector: How to Make the Difference, The Brookings Institution Press Washington, 2012.

12 Disponible en: http://www.opengovernment.org.

13 Idem.

14 Merino, Mauricio, "La transparencia como política pública”, en Ackerman, John (ed.), Más allá del acceso a la información. Transparencia, rendición de cuentas y Estado de derecho, México, Siglo XXI Editores, 2008, pp. 240-262.

15 Disponible en: http://www.oecd.org/gov/46560184.pdf. 
de información según lo mandatan sus leyes, sino que es necesario que la información sea presentada de tal manera que promueva la colaboración intergubernamental ${ }^{16} \mathrm{y}$ la participación ciudadana.

Albert Meijer ${ }^{17}$ apunta que las políticas de transparencia deben ser proactivas, es decir deben dar a las personas tanto visión como voz. Por ello su objetivo debe ser poner a disposición de las personas información pública de manera sencilla, clara y oportuna para que el ciudadano cuente con los elementos necesarios para participar en la toma de decisiones públicas y poder exigir a las autoridades sus derechos, la prestación de servicios públicos, además de contar con las herramientas que le permiten evaluar el que hacer gubernamental. Para lograrlo, los gobiernos cuentan con diferentes alternativas como el uso tradicional de los medios de comunicación, ${ }^{18}$ o las unidades de información en sus dependencias. Sin embargo, el Internet constituye, hoy por hoy, la herramienta más poderosa para lograr la colaboración y participación en las decisiones públicas, el conocimiento de los derechos, así como para la posterior evaluación de sus resultados. Estas herramientas tienen el potencial de poder fomentar la interacción entre los órganos del Estado y la sociedad estableciendo las bases de un gobierno participativo. ${ }^{19} \mathrm{El}$ acceso a la información, como parte del derecho de libertad de expresión, está ligado también con la democracia pues esta se ve fortalecida en la medida que se cuente con un electorado con conocimientos que tome decisiones informadas. ${ }^{20} \mathrm{En}$ realidad son las personas las titulares de la información generada por los órganos del Estado, pues éstos actúan en su nombre, de ahí que tengan el derecho de conocer y utilizar esa información pública para exigir y ejercer sus derechos, incluidos tanto pedir cuentas a las autoridades como participar en las decisiones públicas.

16 Fierro, Ana Elena y J. Ramón Gil-García, El acceso a la..., cit.

17 Meijer, Albert, "Electronic Records Management and Public Accountability", The Information Society, vol. 17, núm. 4, 2001, pp. 259-270.

18 Kenski, Kate y Stroud, Natalie Jomini, Connections Between Internet Use and Political Efficacy, Knowledge-Journal of Broadcasting \& Electronic Media, 50:2, 2006, pp. 173-192.

19 Noveck, Beth Simone, Wiki Government: How Technology Can Make Government Better, Democracy Stronger, and Citizens More Powerful. Brookings, Press, 2009.

20 Abramson, Paul R. y Aldrich, John H., "The Decline of Electoral Participation in America", American Political Science Review, 1982. 
Luego, el objetivo de los portales de transparencia es configurarse como las herramientas que garanticen el acceso a información pública de manera sencilla tanto en su lenguaje como en su formato; que sea oportuna, con datos abiertos que permitan su reutilización, ${ }^{21}$ enfocada a promover el eficaz ejercicio de los derechos, la participación ciudadana en las decisiones públicas, así como en la posterior evaluación de los resultados. De manera que conformen un puente de comunicación ${ }^{22}$ que permita a todas las personas ejercer sus derechos, exigir servicios públicos de calidad ${ }^{23}$ y participar de las decisiones públicas.

\section{Portales De tRANSPARENCIA EN MÉXICO}

Grandes son los esfuerzos que desde principios del siglo XXI México ha llevado a cabo para desarrollar el derecho de acceso a la información pública y el correlativo deber de transparencia. A pesar de que el acceso a la información pública posee rango constitucional desde la Reforma Política de 1977 (sólo como una garantía social exigible por los partidos políticos), ${ }^{24}$ su consolidación como un derecho humano ha sido un camino largo que aún no puede considerarse culminado. Para 1982, con la ratificación por parte del Senado de diversos instrumentos internacionales en materia de derechos humanos ${ }^{25}$ que reconocían el acceso a la información con esta calidad, así como las sucesivas interpretaciones que de este derecho hizo la Suprema Corte de Justicia, se fue avanzando en su desarrollo. Sin embargo, se enfrentaban a obstáculos importantes, en virtud de que no

21 Smith, Alastair G., Applying Evaluation Criteria to New Zealand Government Websites. International Journal of Information Management, vol. 21, núm. 2, 2001, pp. 137-149.

22 Ataloglou, Michail P. y Anastasios, A., Economides, Evaluating European Ministries' Websites, 2007, disponible en: http://grandsorganismes.gouv.qc.ca/upload/cego/ editor.

23 Capgemini, IDC, Rand Europe, Sogeti and DTi. Digitizing Public Services in Europe: Putting ambition into action. European Commission. 9th Benchmark Measurement, 2010; Soonhee, Kim y Lee, Jooho, E-Participation, Transparency, and Trust in Local Government, 2012, disponible en: http://onlinelibrary.wiley.com/doi/10.1111/j.1540-6210. 2012.02593.x/epdf.

24 Semanario Judicial de la Federación, Séptima Época, sexta parte, vols. 145-150, pp. 226 y 227.

25 Véase, supra, nota 1. 
existía una ley reglamentaria del artículo 6o. que estableciera los términos y condiciones en los que la información debiera publicarse ni mucho menos sus características. En 2002 se emite la Ley Federal de Acceso a la Información Pública a la que le siguen sendas leyes estatales. Si bien esto permitió contar con reglas de qué información era de acceso público y procedimientos definidos de cómo solicitarla, así como las primeras regulaciones de qué información debiera ser pública de oficio, sin necesidad de que mediara solicitud alguna, existía una enorme disparidad aún a nivel normativo en la forma, periodicidad y contenido que dicha información pública debiera cumplir. Si el acceso a la información gubernamental es un derecho humano resultaba inadmisible que dentro del país existiera tal diversidad de reglas para su ejercicio.

De ahí que el derecho al acceso a la información sólo adquiere verdadera fuerza cuando es desarrollado mediante la posterior reforma constitucional de 2007 en la que se sientan las bases y principios que debe atender toda autoridad a fin de garantizar el acceso a la información gubernamental de manera uniforme en todo el país. Ello impone a las autoridades el deber de informar en los términos y con las condiciones establecidas en la Constitución. El artículo 6o. ya ordenaba que cierta información debiera estar publicada vía Internet entre la que destacaba los indicadores de gestión. En general se consideraba aún que la información pública de oficio debiera ser aquella que permitiera evaluar la actuación de los sujetos obligados, en particular respecto del uso de los recursos públicos.

La reforma constitucional de $2014^{26}$ busca avanzar en la consolidación del derecho de acceso y establece obligaciones puntuales a todos los sujetos obligados, además de ampliar su regulación a partidos políticos y sindicatos. Respecto del manejo y publicación de la información, el nuevo artículo 6o. constitucional explicita la obligación de toda autoridad de documentar cada uno de sus actos, regirse por el principio de máxima publicidad y restringir la reserva de información a causas de seguridad nacional. En particular, el artículo 6o. en la fracción VI dispone:

Los sujetos obligados deberán preservar sus documentos en archivos administrativos actualizados y publicarán, a través de los medios electrónicos disponibles, la información completa y actualizada sobre el ejercicio de los

26 Publicación en el Diario Oficial de la Federación, 7 de febrero de 2014, disponible en: http://www.dof.gob.mx/nota_detalle.php? codigo $=5332003 \&$ fecha $=07 / 02 / 2014$. 
recursos públicos y los indicadores que permitan rendir cuenta del cumplimiento de sus objetivos y de los resultados obtenidos. ${ }^{27}$

De tal manera que ya la Constitución ordena no sólo mantener archivos actualizados, sino que supone que estos archivos son electrónicos lo que, como señala Albert Meijer, ${ }^{28}$ impone retos a los formatos en que la información se genera, resguarda y publica. En efecto, es importante reconocer que, para esta política de transparencia proactiva, las tecnologías de la información en general, pero sobre todo los recursos como wikis, blogs, portales y redes sociales constituyen sus herramientas principales. ${ }^{29}$ La reforma constitucional al artículo 6o. de 2014 enfatizó estos elementos proactivos al establecer que la obligación de documentar el actuar de los sujetos obligados incluye hacerlo en medios electrónicos. En esta misma línea, la Ley General de Transparencia (LGT) ya incorpora, dentro del título cuarto, capítulos correspondientes a la transparencia proactiva, siguiendo la Ley Modelo Interamericana sobre acceso a la información pública y los lineamientos del AGA. Hoy, la LGT establece para todos los sujetos obligaciones respecto de la calidad de la información pública para garantizar su acceso en formatos abiertos y su posible reutilización.

Asimismo, el inciso B del artículo 6o. en su fracción I señala que: “el Estado garantizará a la población su integración a la sociedad de la información y el conocimiento"; ${ }^{30}$ por tanto, desde la Constitución parece reconocerse la importancia del Internet en general y de los portales de transparencia en particular como las herramientas que permitan esta comunicación entre personas y sujetos obligados que garantice el derecho de acceso a información pública de manera sencilla tanto en su lenguaje como en su formato, oportuna, con datos abiertos que permiten su reutilización, enfocada a promover el conocimiento y ejercicio de los derechos, así como la participación ciudadana en las decisiones públicas y la posterior evaluación de los resultados.

27 Constitución Política de los Estados Unidos Mexicanos, última reforma publicada en el Diario Oficial de la Federación, 27 de enero de 2016, disponible en: http://www. diputados.gob.mx/LeyesBiblio/ref/cpeum.htm.

28 Meijer, Albert, Electronic Records Management..., cit.

29 Fierro, Ana Elena y Gil-García, J. Ramón, El acceso a la información..., cit.

30 Ley General de Transparencia y Acceso a la Información Pública, disponible en: http://www.dof.gob.mx/nota_detalle.php? codigo $=5391143 \&$ fecha $=04 / 05 / 2015$. 
De tal manera que a partir del AGA y conforme a los nuevos parámetros establecidos por el artículo 6o. constitucional, en realidad afrontamos el reto de generar una política de transparencia proactiva. Esta política implica una forma de reorganización gubernamental que busca acercar en la toma decisiones a todos los agentes mediante la generación $\mathrm{y}$ uso de información compartida. De tal manera que el derecho de acceso a la información se convierte en instrumento para conocer y ejercer nuestros derechos, así como el primer paso del derecho a la participación en la toma de decisiones. Los portales de transparencia deben ser la ventana que permita la comunicación y colaboración entre gobernantes y gobernados.

En contraste con lo hasta aquí apuntado, lo cierto es que los portales de transparencia en México se han concentrado en un mero cumplimiento de jure, es decir, en usar el portal como un receptáculo de la información que establece la Ley de Transparencia sin preocupación alguna por su utilidad o por intentar conocer qué información y en qué formatos en realidad es útil para el ciudadano. ${ }^{31}$ La dimensión de información pública de oficio de Métrica de Transparencia 2014, ${ }^{32}$ así lo demuestra. La calificación respecto al cumplimiento formal de presentar información que requiere la ley respectiva más alta fue de 0.98 obtenida por el Distrito Federal, seguido por Zacatecas que obtuvo 0.96. La calificación más baja fue de 0.63 para Baja California Sur. Al igual que en la Métrica 2010, ${ }^{33}$ los resultados de esta medición indican (IPO) no está relacionada ni con el nivel de gobierno, ni con el poder al que pertenece.

Ahora bien, si comparamos los resultados de la Métrica 2010 con los de 2014 en materia de la regulación de la información pública de oficio en general, se percibe un aumento importante en el catálogo de la IPO establecida en las leyes. Sin embargo, este aumento por sí solo no implica que los portales de transparencia permitan en realidad dar conocer más información pública. Es decir, si bien es deseable y de hecho mejora la calificación de un portal el tener más información, ello no basta para que sea de los mejores portales de transparencia, sino que se requiere que la

31 Cucciniello, María y Nasi, Greta, International Journal of ..., cit.

32 Para una explicación detallada de la metodología seguida en la evaluación de los portales de transparencia, disponible en: http://www.metricadetransparencia.cide.edu/?s ection=DimensionesPortales.

33 Disponible en: http: //www.metricadetransparencia.cide.edu/?section=Dimensio nesPortales, Grafica 1. Subíndice portales. 
información tenga calidad, orden y oportunidad. Además de contar con la tecnología necesaria para soportar toda la información y facilitar su búsqueda. De no cumplirse con lo anterior se corre el riesgo de que las personas no encuentren la información requerida o que esté en un formato que no le permite reutilizarla. Los casos paradigmáticos de ello se muestran en la siguiente:

Tabla 1. IPO en las leyes de transparencia

\begin{tabular}{|c|c|c|c|c|}
\hline Estado & $\begin{array}{c}\text { IPO en Ley de } \\
\text { Transparencia } \\
2010\end{array}$ & $\begin{array}{c}\text { IPO en Ley de } \\
\text { Transparencia } \\
2014\end{array}$ & $\begin{array}{c}\text { Calificación } \\
\text { de portales de } \\
\text { transparencia } \\
2010\end{array}$ & $\begin{array}{c}\text { Calificación } \\
\text { de portales de } \\
\text { transparencia } \\
2014\end{array}$ \\
\hline Jalisco & 94 & 165 & 0.85 & 0.94 \\
\hline Coahuila & 67 & 104 & 0.76 & 0.87 \\
\hline Veracruz & 83 & 102 & 0.77 & 0.82 \\
\hline
\end{tabular}

Fuente: Métrica de la Transparencia 2014. ${ }^{34}$

Otro ejemplo en el que si bien hay avances, éstos se limitan a un cumplimiento de jure es el relativo a los indicadores cuya publicación ordena la Constitución desde la reforma de 2007. En la Métrica 2010 se reportó que la información respecto a indicadores de gestión mandatada por el artículo 6o. fracción VI vigente era prácticamente inexistente. En contraste, la Métrica 2014 registra que 22 leyes de transparencia ya contemplan a los indicadores como IPO, lo que es un avance importante. Sin embargo, existe gran heterogeneidad en cómo se reportan.

34 Disponible en: http://www.metricadetransparencia.cide.edu. 


\section{Gráfica 1. Indicadores de gestión}

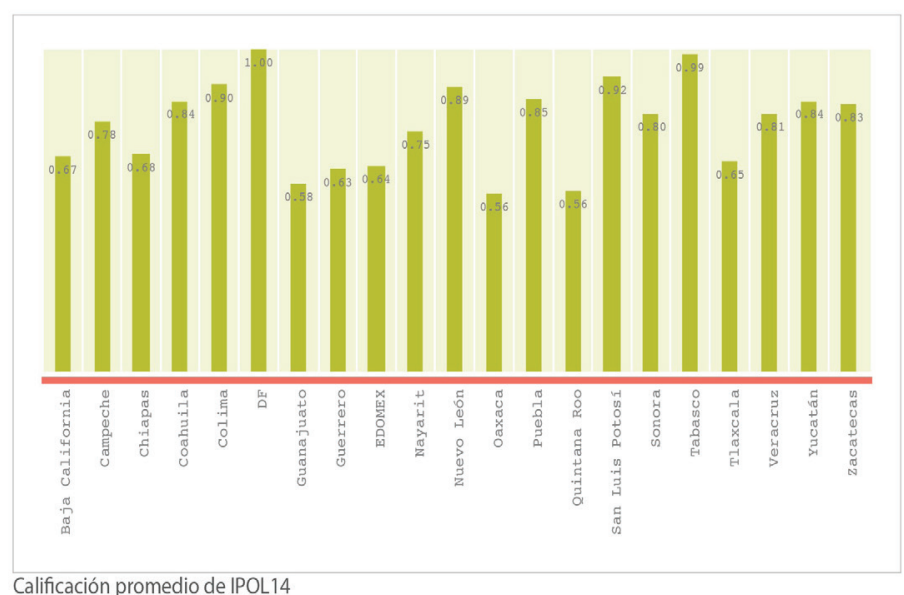

Calificación promedio de IPOL14

FuENTE: Métrica de la Transparencia 2014. ${ }^{35}$

De nueva cuenta se trata de un cumplimiento de jure sin que haya una preocupación por la utilidad real de la información respecto de los indicadores, ni la posibilidad de que ésta pueda ser utilizada para el ejercicio de facto de los derechos de acceso a la información pública y exigencia de rendición de cuentas. Nada de esta información se encuentra en formatos de datos abiertos, no aparece la metodología con la que estos indicadores son creados y por supuesto no es posible compararlos.

La reforma al artículo 6o. de 2014 ha dado gran relevancia a este punto pues establece que los indicadores deben permitir rendir cuenta de los resultados obtenidos. La situación actual es de una heterogeneidad preocupante ya que aún no hay consenso sobre qué se entiende por indicadores de gestión y quiénes deben reportarlos. Por ejemplo, a pesar de que el artículo 6o. constitucional señala que es obligación de todos los sujetos obligados publicar sus indicadores, en la Ley de Transparencia de Tamaulipas, ${ }^{36}$ se establece que es una obligación exclusiva del Poder Ejecutivo. Otro problema sobre este mismo aspecto es que los indicadores generalmente

35 Idem.

36 Ley de Transparencia y Acceso a la Información Pública del Estado de Tamaulipas, disponible en: http://transparencia.tamaulipas.gob.mx/wp-content/uploads/2014/03/TFI-LEY-DE-TRANSPARENCIA-Y-ACCESO-A-LA-INFO-PUBLICA-23052013.pdf. 
se presentan en formato PDF y sin establecer la metodología con que se desarrollaron.

De tal manera que si bien es cierto que en la actualidad podemos decir que la mayoría los sujetos obligados cuentan con un portal de transparencia y un mecanismo electrónico para presentar solicitudes de acceso a la información y que las leyes de transparencia se han preocupado por ampliar el catálogo de IPO, el esfuerzo ha quedado limitado a un cumplimiento de jure, sin preocupación alguna por la calidad o utilidad de la información publicada que pudiera asegurar el cumplimiento de facto.

En efecto, la Métrica 2014 demuestra que actualmente presentar información pública de oficio a través de los portales de transparencia es una labor incorporada a las rutinas de los sujetos obligados. Sin embargo, al igual que en la Métrica 2010 prevalece un problema importante de heterogeneidad de la IPO presentada en los portales y de su baja calidad. ${ }^{37} \mathrm{De}$ hecho, el aumento del catálogo de la IPO en las leyes de transparencia de las entidades federativas agravó la disparidad de la información entre los distintos sujetos obligados y redujo de forma importante su comparabilidad. Por ejemplo, las variables comunes en las legislaciones de los estados que permitían la comparabilidad entre entidades en la Métrica 2010 eran 13. Debido a los cambios normativos ocurridos en los años posteriores, en la Métrica 2014 sólo se identificaron cinco variables totalmente transversales para todos los sujetos obligados, en todas las legislaciones del país.

Tabla 2. Comparativo de IPO común 2010 y 2014

\begin{tabular}{|l|l|}
\hline \multicolumn{1}{|c|}{ IPO común M2010 } & \multicolumn{1}{|c|}{ IPO común M2014 } \\
\hline Marco normativo & Marco normativo \\
\hline Estructura orgánica & Estructura orgánica \\
\hline Directorio de servidores & Directorio de servidores \\
\hline Trámites y servicios & Trámites y servicios \\
\hline Programas operativos & Programas operativos \\
\hline Datos de la unidad de enlace & \\
\hline Información presupuestal & \\
\hline Avance del presupuesto & \\
\hline
\end{tabular}

37 Disponible en: http://www.metricadetransparencia.cide.edu. 


\begin{tabular}{|l|l|}
\hline Tabulador de remuneraciones & - \\
\hline Contratos & - \\
\hline Informe de actividades & - \\
\hline Apoyos y subsidios & - \\
\hline Resultado de las auditorías & - \\
\hline
\end{tabular}

FUENTE: Métrica de la transparencia 2014. ${ }^{38}$

De lo anterior no debe colegirse que la información respecto de presupuesto, remuneraciones o contratos no exista en algunas leyes, sino que la manera en que las normas han desagregado esta información y a los sujetos a los que han obligado a publicarla varía de manera importante, por lo que su publicación como IPO en los portales es diferente, lo que dificulta su comparabilidad. Por ejemplo, la variable relativa al avance del presupuesto común a todos en 2010 no aparece en la legislación de Chiapas. ${ }^{39}$ En esta entidad existen otras variables que muestran información sobre el uso de los recursos públicos que se refiere a constituir fondos locales que se incluyan al presupuesto de las mismas, o que se refiere a la cuenta pública. Como se puede apreciar, este tipo de variables no permiten una comparación exacta. Otro ejemplo de esta problemática es la diversidad de regulación en lo que se refiere a contratos.

Esta heterogeneidad se ve agravada porque el catálogo de IPO de las Leyes de Transparencia se hace sin tomar en cuenta la organización de las administraciones públicas de las entidades federativas. Esta omisión produce que los sujetos obligados argumenten que muchas de las fracciones relativas a la IPO no les aplican dado que Ley Orgánica de la Administración Pública de su estado asigna esa función en competencia exclusiva a un determinado sujeto obligado o incluso afirman que es competencia de otro poder. Tales son los casos de la IPO relacionada con deuda pública, obra pública, padrón de proveedores y de la cuenta pública. ${ }^{40}$

El caso más común es el de la cuenta pública, pues los sujetos obligados con frecuencia afirman que "No Aplica" a pesar de que las leyes de transparencia en general la definen como una obligación para todos los sujetos

\footnotetext{
38 Disponible en: http://www.metricadetransparencia.cide.edu.

39 Idem.

40 Disponible en: $h t t p: / / w w w . m e t r i c a d e t r a n s p a r e n c i a . c i d e . e d u / ?$ section=Dimension esPortales.
} 
obligados. La interpretación formulada por la administración pública local suele ser que dar esa información compete a las entidades de fiscalización superior exclusivamente. ${ }^{41} \mathrm{Si}$ esto es así, al menos valdría la pena que la fundamentación y motivación correspondiente apareciera en el portal. Sin embargo, pareciera que si el legislador estableció como IPO para todo sujeto obligado la cuenta pública se refiere al reporte de cuenta pública que cada sujeto debe presentar a la auditoría superior competente (federal si son recursos federales, o local si éste es el origen de los recursos públicos) y no al resultado del análisis del mismo que corresponde en exclusiva a los órganos de fiscalización. De cualquier forma, se reitera que, si bien la organización interna de cada uno de los poderes y de la administración pública corresponde a cada estado, lo importante es que al menos las personas pudieran tener claras las razones por las cuales no aparece información en determinado portal.

Además, esta discrepancia entre las leyes de transparencia y las que organizan a la administración pública - o a los otros poderes- parecen obligar al ciudadano a conocer toda la regulación administrativa del Estado para poder encontrar la información que necesita. Lo cierto es que estas discrepancias al interior del ordenamiento jurídico de la entidad junto con el abuso de "No Aplica" resultan en opacidad, pues imponen altas barreras para lograr acceder a la información pública.

En efecto, esta lógica burocrática limitada a un mero cumplimiento de jure del catálogo de IPO establecido en la Ley, resulta una mala práctica, pues termina por no dar acceso a IPO y sin duda no se preocupa por las necesidades de las personas con respecto a la información que requiere para el adecuado ejercicio de sus derechos, y menos aún por fomentar la participación ciudadana. En algunos casos incluso se busca justificar por la estructura orgánica o el grado de autonomía de cierto sujeto obligado prácticamente su exclusión de las obligaciones de transparencia. Tal es el caso, por ejemplo, de la Secretaría de Salud de Jalisco. En 16 variables que, conforme con la Ley de Transparencia de Jalisco, son obligaciones para todos los sujetos, afirma que no le aplican porque esas funciones corresponden a un recién creado órgano público descentralizado de servicios de salud. ${ }^{42}$ Si bien esta respuesta se apega a la legalidad formal, en la búsqueda de información relativa a las obligaciones relacionadas con la protección del

\footnotetext{
41 Idem.

42 Reporte de portales. Métrica de transparencia, México, CIDE, 2014.
} 
derecho humano a la salud lo único que se encuentra es una justificación amparada en distribución de competencias entre órganos del Estado y de facultades concurrentes, que de poco o nada sirve.

También resulta preocupante que los sujetos obligados desarrollen justificaciones legales amparadas en legislaciones diversas a la Ley de Transparencia para no otorgar la información. Un ejemplo de ello es el caso de la obligación establecida por la Ley de Transparencia de Jalisco de hacer públicas las declaraciones patrimoniales, a lo que los sujetos obligados responden que la Ley de Responsabilidad de Servidores Públicos del Estado exige el consentimiento de los servidores para publicar su declaración patrimonial y que como un grupo de servidores públicos habían promovido un amparo y se les había concedido la suspensión, la autoridad había decidido no publicar ninguna declaración patrimonial. ${ }^{43}$ De nueva cuenta se trata de una respuesta legalista que incluso pudiera cuestionarse, pues se entiende que sólo algunos servidores públicos promovieron el juicio de amparo, por lo que en realidad los beneficios de la suspensión sólo protegen a los quejosos y no a todos los servidores públicos obligados a presentar su declaración patrimonial y hacerla pública. Si bien es cierto que se trata de un caso controvertido, dado que la declaración patrimonial contiene datos personales y pudiera significar riesgos de seguridad para los funcionarios y sus familias, la mera justificación procesal resulta insuficiente. Este ejemplo muestra cómo los portales de transparencia en México tampoco logran ser una herramienta eficaz para ejercer a cabalidad nuestros derechos, recibir servicios públicos, exigir cuentas ni participar en las decisiones públicas.

Similares consecuencias resultan de las malas prácticas en la publicación de los perfiles de puestos. Si la Ley de Transparencia señala que el sujeto obligado debe publicar el perfil de puestos de sus directivos, no basta con que en el portal aparezca el listado de los puestos. El perfil de puesto se refiere a las funciones esenciales y la responsabilidad de cada cargo dentro del sujeto obligado. La definición de puestos se basa en conocer los requisitos y cualificaciones personales exigidos para un cumplimiento satisfactorio de las tareas correspondientes a ese puesto, como puede ser el nivel de estudios o la experiencia laboral requerida. ${ }^{44}$ De nueva cuenta este

\footnotetext{
43 Idem.

44 Disponible en http://www.armstrong.com.mx/consultoria-organizacional/definicion-de-perfiles-de-puesto.php.
} 
rubro muestra cómo se agrega a los portales de transparencia documentos de muy poca utilidad con el solo fin de cubrir formalmente las obligaciones de IPO que señala la ley correspondiente.

Sin duda una de las prácticas más perniciosas respecto a la calidad de la información presentada en los portales de transparencia es el uso proliferado de documentos en PDF principalmente en lo que se refiere a la información correspondiente al resultado de las auditorías y el ejercicio del presupuesto. Este formato limita el acceso a la información e impide que se pueda reutilizar. La práctica internacional, promovida por el AGA, señala que la información relativa al uso de los recursos públicos debe estar en formatos de datos abiertos, es decir en bases de datos fácilmente manipulables. Hoy, la LGT en su artículo 59 ya lo establece como una obligación de todos los sujetos obligados. Desafortunadamente ésta no es una práctica de los portales de transparencia mexicanos.

Del análisis de los portales de transparencia se desprende que en la publicación de IPO se ha caído en la trampa del procedimiento que señala Mauricio Merino: ${ }^{45}$ los portales están conformados con una lógica de cumplimiento normativo de jure en el que una parte importante de la información no se publica de manera clara ni con formas de acceso rápido o se publica parcialmente bajo justificaciones legalistas. Hay muy poco esfuerzo por hacer la información accesible, se utiliza un lenguaje legal rebuscado, lleno de tecnicismos sin preocupación alguna por que lo comprenda todo tipo de personas. En la mayor parte de la información hay un uso generalizado de formatos que impiden la reutilización de la IPO. Esta lógica estricta de mero cumplimiento de jure denota la falta de una política de transparencia proactiva que en realidad permita el uso estratégico de la información pública.

Hoy en día los portales de transparencia no son herramientas que garantizan el acceso a información pública de manera sencilla, sino meros formatos donde cumplir formalmente con la ley. Este uso limitado de los portales de transparencia resulta en deficiencias importantes en el ejercicio del derecho de acceso a la información pública.

\footnotetext{
45 Mérino, Mauricio, "La transparencia como política pública", cit.
} 


\section{RETOS Y OPORTUNIDADES}

Hoy en México la transparencia proactiva no puede ser vista como un ejercicio gracioso de la autoridad; es un mandato constitucional y parte de las obligaciones internacionales adquiridas por el Estado mexicano a través del AGA. En consecuencia, es importante que tanto a través de la LGT como con la subsecuente legislación secundaria a nivel federal y local, se regule no sólo el catálogo de la IPO, sino también la calidad de la información presentada en los portales de transparencia, de modo que se sienten las bases de una política pública de transparencia, que asegure el goce cabal del derecho de acceso a la información.

El punto de partida para mejorar la IPO disponible tendría que ser definir cuáles son los objetivos de los portales de transparencia. Si éstos constituirán junto con la Plataforma Nacional de Transparencia, regulada en los artículos 49 a 52 de la LGT, el vehículo de implementación de una política de transparencia proactiva.

Para lograr de facto el ejercicio del derecho de acceso a la información es necesario preguntarse qué información de cada sujeto obligado resulta útil como IPO y en qué formatos debe presentarse. Aunque el artículo 6o. constitucional señala que toda información que resulte de "documentar todo acto que derive del ejercicio de facultades, competencias o funciones ${ }^{\prime \prime 4}$ de los sujetos obligados debe ser accesible, quizás no para todos los sujetos obligados es viable ofrecerlo dentro de los portales de transparencia. Lo que habrá que buscar es que los portales sean vasos comunicantes entre las personas y los sujetos obligados donde el énfasis se encuentre en aquella información que es útil para ejercer sus derechos, incluyendo los de participar de las decisiones públicas y exigirles cuentas a las autoridades. De ahí que parece deseable en la normatividad federal y local, que aún está pendiente, buscar un equilibrio entre la IPO y el acceso a través de procedimientos de acceso a la información cada vez más simples y rápidos, además de cumplir con las buenas prácticas en cuanto a lenguaje claro, oportunidad, claridad y datos abiertos.

Es indispensable tener en cuenta que ahora con la LGT ya no será suficiente con tener portales que publiquen IPO con el fin exclusivo de cum-

46 Constitución Política de los Estados Unidos Mexicanos, título primero, capítulo I, "De los derechos humanos y sus garantías" (cambio de denominación mediante decreto publicado en el Diario Oficial de la Federación del 10 de junio de 2011, artículo 6o.). 
plir con la transparencia de jure, pues será obligación de todos los sujetos contar con una política de transparencia proactiva y estrategias de gobierno abierto. Esto implica adoptar medidas que garantizan la calidad de la información en cuanto acceso y utilización, es decir una transparencia de facto.

Asimismo, la LGT establece un conjunto de obligaciones para los órganos garantes tendentes a coordinar una política de transparencia proactiva que incorpore las buenas prácticas en materia de gobierno abierto. Estas nuevas obligaciones significarán retos importantes para todos los sujetos obligados tanto por lo que se refiere a la regulación en la legislación local, como en su implementación a través del Sistema de Nacional de Transparencia, contemplado en el artículo 6o. constitucional.

Ahora bien, la LGT señala un extenso catálogo de IPO. Establece, en su artículo 70, 48 supuestos de IPO comunes a todos los sujetos obligados y en artículos subsecuentes 88 obligaciones adicionales por tipo de sujeto obligado. ${ }^{47}$ Como muestran los resultados de la Métrica de Transparencia 2014, estos catálogos extensos de IPO no conllevan necesariamente a portales que permitan mayor acceso a información pública y menos a ser vasos de comunicación y uso de la información por parte de las personas. Por tal motivo, es necesario atender en la implementación de la LGT a las particularidades de la entidad federativa y asegurar la calidad de la información. En este sentido resultará necesario que las leyes de transparencia federal y local establezcan con claridad el objetivo de cada portal y regulen atendiendo a las competencias específicas de cada entidad federativa quiénes, cómo y con qué temporalidad presentarán la IPO a partir de su propia organización competencial.

A fin de evitar la práctica de señalar que "No Aplica" tal o cual obligación de IPO a determinado sujeto obligado las nuevas leyes de transparencia local y federal, deben adecuar estas obligaciones de IPO de la LGT a su ordenamiento jurídico, de manera que, atendiendo a las competencias y facultades realmente asignadas a cada uno de los sujetos obligados, señale la IPO que efectivamente le corresponda. Es evidente que los estados, como niveles de gobierno autónomo y soberanos en el marco de la Constitución, pueden determinar la organización interna de sus poderes; sin embargo, será esencial que las leyes de transparencia de cada entidad federativa al momento de reglamentar la LGT tengan en cuenta estas particularidades

47 Ley General de Transparencia y Acceso a la Información Pública, disponible en: http://www.dof.gob.mx/nota_detalle.php? codigo $=5391143 \&$ fecha $=04 / 05 / 2015$. 
propias de manera que del catálogo común asignen las obligaciones de publicación de IPO a los sujetos que efectivamente tengan las competencias correspondientes a dichas obligaciones. Sobre todo, deberán ordenar que la información publicada cuente con las características necesarias para realmente responder a las necesidades de las personas.

El artículo 6o. constitucional hoy en día establece la obligación de publicar en los portales de transparencia los indicadores de resultados para rendir cuentas. La LGT regula de manera detallada esta obligación. Por un lado, dentro de las obligaciones del Sistema Nacional del Transparencia se encuentra la de establecer las metodologías para la creación de los indicadores. Por el otro, dentro de la IPO general (artículo 70, fracciones VI y XV) se regula la publicación de indicadores estratégicos, de gestión y para rendir cuentas de los resultados obtenidos y tratándose de programas de subsidios; además, debe publicarse nombre, definición, método de cálculo, unidad de medida, dimensión, frecuencia de medición y nombre de las bases de datos utilizadas para su cálculo. Con esta regulación se busca abatir la heterogeneidad que los indicadores de gestión presentan en la actualidad, de manera que realmente sea posible comparar los resultados de la gestión de los diversos sujetos obligados y evaluar el cumplimiento de sus objetivos, fortaleciendo con ello a los mecanismos de rendición de cuentas.

Respecto a la calidad de la información, la LGT adopta las prácticas internacionales. Establece dentro de los principios que deben regir la publicación el de accesibilidad. Fija la obligación del Sistema Nacional de Transparencia de crear los formatos de publicación de la información para asegurar que sea veraz, confiable, oportuna, congruente, integral, actualizada, accesible, comprensible, verificable y en formatos abiertos. Además, la LGT indica la obligación de que la IPO sea presentada en formatos que permitan el acceso a grupos vulnerables, como indígenas y discapacitados. También promueve el uso de datos abiertos. Sin embargo, para lograrlo es necesario que los sujetos obligados superen la lógica de un mero cumplimiento formal de la ley en la que ahora se encuentran y entiendan que los portales de transparencia pueden ser una gran herramienta que les permita implementar una política de transparencia proactiva, tendente a asegurar, como les mandata el párrafo 3 del artículo 1o. constitucional, la promoción y garantía de los derechos humanos. Para ello, los portales de transparencia deberán responder a las mejores prácticas respecto de la calidad de la información. 
De acuerdo con los lineamientos de AGA y la práctica internacional toda información pública debe estar presentada con lenguaje claro y si es necesario en lenguas indígenas o con mecanismos para personas con capacidades diferentes; además tener niveles de especialización para públicos diversos, formatos de datos abiertos, proveer trámites y servicios en línea que faciliten el acceso a los servicios público y el ejercicio de los derechos, mecanismos de comunicación con los usuarios para que levanten quejas o sus dudas sean desahogadas. Además, la información de los portales debe ser indexable por motores de búsqueda, con mapas del sitio, enlaces identificables y acceso libre (sin derechos de autor).

\section{REFLEXIONES FINALES}

La reforma al artículo 6o. constitucional de 2014 y la LGT han puesto las bases para el ejercicio del derecho de acceso a la información pública, lo que sin duda es un avance importante. Sin embargo, esta es la parte sencilla; hacer realidad la transparencia proactiva como garantía de acceso a la información pública para todos, es la meta. La implementación de la reforma constitucional implica un reto enorme que requiere de esfuerzos y voluntad para realmente entender a la información como un bien público propiedad de las personas y no de las autoridades.

El nuevo marco constitucional abre la oportunidad para transitar de portales de transparencia limitados al cumplimiento de obligaciones legales, es decir de una transparencia de jure a la conformación de una política de transparencia proactiva que permita un acceso a la información pública que en verdad fomente la colaboración intergubernamental, el ejercicio de los derechos y la participación ciudadana. La política de transparencia proactiva debe asegurar que los ciudadanos puedan conocer la actuación de todos los sujetos obligados de manera accesible y que puedan comparar y reusar la información con el objetivo de hacer efectivo el ejercicio de sus derechos, facilitar la participación.

Los portales de transparencia y la información en ellos contenida deben, ante todo, servir a las personas para ejercer todos sus derechos. Ello implica dejar de utilizarlos como meros receptáculos de información para cumplir con la ley y convertirlos en un vehículo de comunicación para la colaboración y la participación ciudadana informada. Aún queda un largo trecho por recorrer, para pasar de un mero cumplimiento de la ley a lograr 
que el derecho humano de acceso a la información, como lo contempla la Constitución, sea una realidad. Lograr portales de transparencia que cumplan con las buenas prácticas aquí enlistadas significará un avance importante en la transformación de la transparencia de jure - actualmente existente - a una política proactiva que asegure de facto el cabal ejercicio del derecho de acceso a la información pública.

\section{BIBLIOGRAFÍA}

Abramson, Paul R. y Aldrich, John H., "The Decline of Electoral Participation in America", American Political Science Review, 1982.

Ataloglou, Michail P. y Anastasios, A., Economides, Evaluating European Ministries, 2007, disponible en: http://grandsorganismes.gouv. qc.ca/upload/cego/editor.

Bauhr, Monica y Grimes, Marcia, What is Government Transparency?, Quality of Government Institute, working paper series, 2012, 16.

CAPgemini, IDC, Rand Europe, Sogeti and DTi. Digitizing Public Services in Europe: Putting ambition into action, European Commission. 9th Benchmark Measurement, 2010.

CIDE, Métrica de transparencia 2010, México, CIDE-Conferencia Mexicana para el Acceso a la Información Pública (COMAIP), disponible en: www.metricatransparencia.cide.edu.

CIDH, Informe Anual, 2000-01, vol. III.

CuCCINIELlo M. et al., "Making transparency transparent: an assessment model for local governments", en Tria, G. y VAlOtTI, G. (eds.), Reforming the Public Sector: How to make the difference, Washington, The Brookings Institution Press, 2012.

Fierro, Ana Elena y GiL-GarcíA, J. Ramón, "Más allá del acceso a la información. El uso de tecnologías de información para fomentar la transparencia, la participación y la colaboración en el sector público", en Cesudo, Guillermo M. et al. (eds.), La politica de transparencia en México, México, CIDE, 2012.

Kraemer, Kenneth L. y King, John Leslie, "Information technology and administrative reform: will e-government be different?", International journal of electronic government research, vol. 2, núm. 1, 2006. 
Kenski, Kate y Stroud, Natalie Jomini, "Connections between Internet Use and Political Efficacy, Knowledge, and Participation", Journal of Broadcasting \& Electronic Media, 2006.

Law, David y Versteeg, Mila Sham, Constitutions, 101 Cal. L. Rev. 863, 2013, disponible en: http://scholarship.law.berkeley.edu/californialaw review/vollo1/iss4/.

LóPEZ Ayllón, Sergio, El acceso a la información como un derecho fundamental: la reforma al artículo 6o. de la Constitución Mexicana, México, Instituto Federal de Acceso a la Información Pública, 2009.

Merino, Mauricio, "La transparencia como política pública", en ACKerMAN, John (ed.), Más allá del acceso a la información. Transparencia, rendición de cuentas y estado de derecho, México, Siglo XXI Editores, 2008.

Noveck, Beth Simone, Wiki Government: How Technology Can Make Government Better, Democracy Stronger, and Citizens More Powerful, Brookings Press, 2009.

Rosendorff, Peter et al., Measuring Transparency, Department of Political Science, University of Minnesota, 2013, disponible en: http:// papers.ssrn.com/sol3/papers.cfm?abstract_id=2113665.

Schnackenberg, Andrew, Measuring Transparency: Towards a Greater Understanding of Systemic Transparence and Accountability, 2002.

SмiтH, Alastair G., "Applying Evaluation Criteria to New Zealand Government Websites", International Journal of Information Management, vol. 21, núm. 2, 2001.

Fecha de recepción: 3 de febrero de 2016.

Fecha de dictamen: 17 de abril de 2016. 